\title{
Medizinisches „Disaster Management“" am Flughafen Frankfurt Strategien und Konzepte bei einem Massenanfall von Verletzten sowie bei importierten Infektionskrankheiten
}

\author{
W. Gaber, Leiter Medizinische Dienste Fraport AG, Flughafen Frankfurt
}

NOTFALLMEDIZIN 2003; 29: 213-215

\begin{abstract}
Die Fraport AG als Betreiberin des Flughafens ist seitens der medizinischen Versorgung bestens auf die unterschiedlichen Schadensereignisse vorbereitet; dennoch müssen sich alle Verantwortlichen (Aufsichtsbehörden, Politiker, Verbandsgemeinden u. a. m.) darüber im „klaren“ sein, dass weder die Fraport, noch die Branddirektion und sonstige Hilfsorganisationen alleine ein großes Schadensereignis wie einen Flugzeugcrash mit bis zu 100 Schwerverletzten „handeln“ können.

Kontinuierliches, gemeinsames Training und „Stop“ von Stellenabbau beziehungsweise Abbau von Transportkapazitäten muss seitens der politisch Verantwortlichen überregional sichergestellt werden.

Die umliegenden Verbandsgemeinden müssen sich offensiv mit der Möglichkeit eines möglichen „Disasters“ auseinandersetzen, Konzepte erstellen und diese kontinuierlich auf ihre Wirksamkeit überprüfen.
\end{abstract}

A lle nationalen und internationalen Verkehrsflughäfen unterliegen nationalen (Frankfurt: Genehmigungsurkunde des Hessischen Ministeriums für Wirtschaft, Verkehr und Landesentwicklung, HMWVL) und internationalen Auflagen (ICAO, International Civil Aviation Organisation) zu personellen und materiellen Vorhaltungen. Die Forderungen gemäß dieser Auflagen reduzieren sich national und international auf das Vorhandensein einer „Pflasterbude“ und einem Doktor „on call“. Die Verantwortung für den Bereich Frankfurt, einschließlich Flughafen, liegt rettungsdienstlich bei der Branddirektion Frankfurt am Main.

Besonderheiten eines internationalen Großflughafens

Die Fraport AG (ehemals FAG) ist grundsätzlich unter Beachtung der behördlichen Aufgaben und Zuständigkeiten für Notfallereignisse und deren Bewältigung auf dem Flughafen verantwortlich und hat entsprechende organisatorische, personelle und materielle Vorkehrungen zu treffen.

Die rettungsdienstliche und notärztliche Verantwortlichkeit liegt bei der Branddirektion Frankfurt/ Main. Dessen ungeachtet werden seitens Fraport qualifizierte Notärzte (Leitende Notärzte Fraport) und Rettungsassistenten (ORLD Fraport) sowie medizinisches Equipment für Großschadensereignisse vorgehalten. Seitens Fraport sind alle Ärzte der „Flughafenklinik“ als Leitende Notärzte (LNA) Fraport qualifiziert. Alle Rettungsassistenten sind, beziehungsweise werden in 2003, als Organisatorischer Leiter Rettungsdienst (ORLD) Fraport qualifiziert. Zielsetzung ist hierbei der nahtlose Übergang und Übergabe sowie Unterstützung des LNA und ORLD Frankfurt. Nach formaler Übergabe an die zuständigen Kollegen werden die Experten von Fraport als Support für die Führungskräfte zur Verfügung stehen.

Im Dreischichtbetrieb stehen 33-2 Rettungswagen (RTW) - alle nach DIN als Notarztwagen (NAW) ausgerüstet - sowie ein bis acht Notärzte von Fraport binnen weniger Minuten via Swissphone während der Regelarbeitszeit zur Verfügung.

Erweitert wird diese Präsenz durch Kooperationen mit dem medizinischen Dienst der DLH (Deutsche Lufthansa), des Bundesgrenzschutzes (BGS) und der US Airforce. Seit 2001 kann der medizinische Dienst auf einen „Disaster Truck“ zurückgreifen, welcher Material für bis zu 180 Patienten vorhält und über zwei aufblasbare Zelte, Licht und eine eigene Stromversorgung verfügt. 

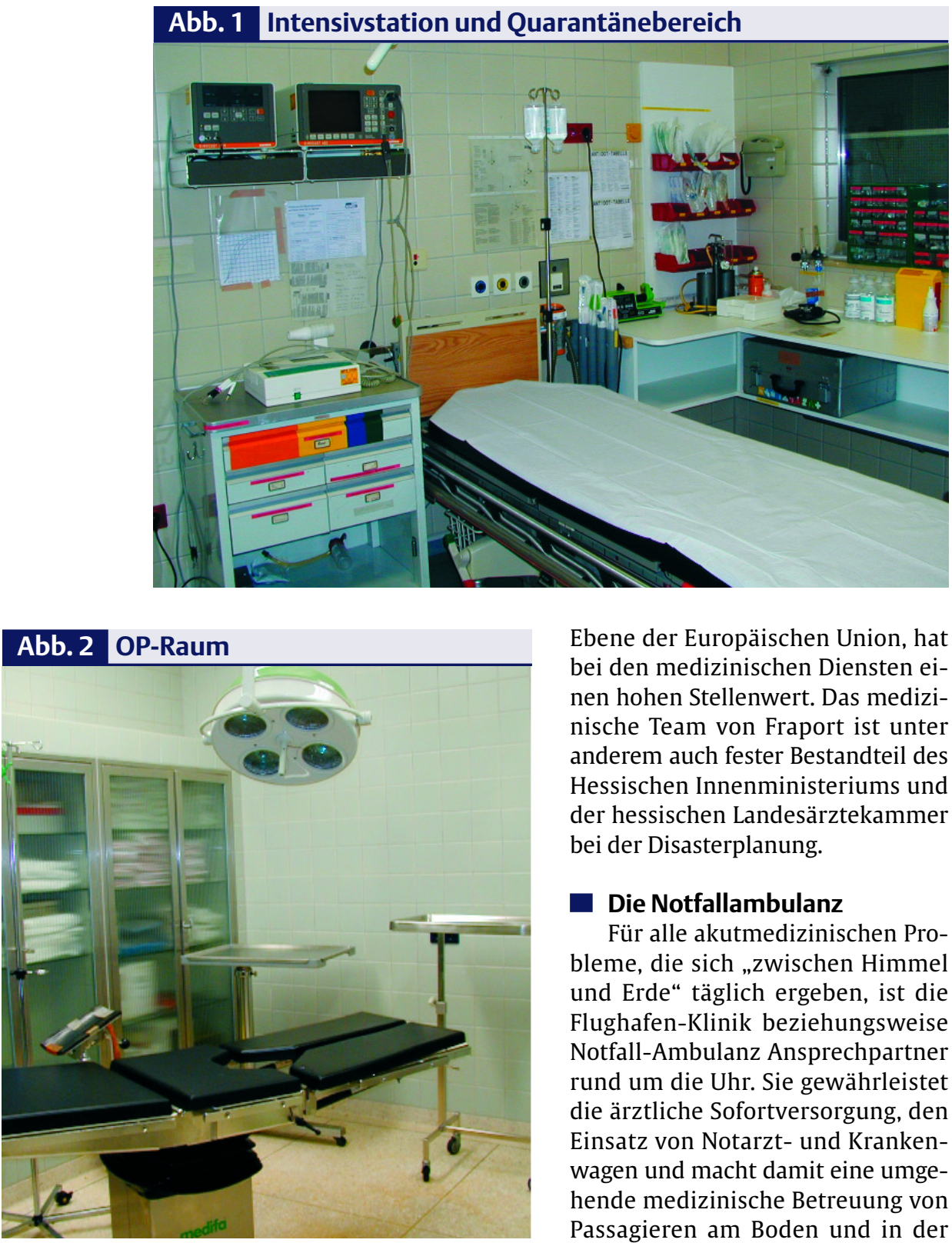

Die Medizinischen Dienste

Die Fraport-AG hat ein umfangreiches Programm zur kompetenten medizinischen Betreuung der Patienten entwickelt und stellt den Kunden ein „Komplettpaket“ in den Bereichen Notfallambulanz, Rettungsdienst und Arbeitsmedizin zur Verfügung. Die medizinischen Dienste von Fraport sind national wie auch international führend im Bereich Medical-Consulting für Flughäfen (Lima, Manila, Antalya, Wien, u.a.). Weiterbildung und Teilnahme als Referenten an internationalen und nationalen Kongressen und Workshops, beispielsweise auf
Ebene der Europäischen Union, hat bei den medizinischen Diensten einen hohen Stellenwert. Das medizinische Team von Fraport ist unter anderem auch fester Bestandteil des Hessischen Innenministeriums und der hessischen Landesärztekammer bei der Disasterplanung.

\section{Die Notfallambulanz}

Für alle akutmedizinischen Probleme, die sich „zwischen Himmel und Erde" täglich ergeben, ist die Flughafen-Klinik beziehungsweise Notfall-Ambulanz Ansprechpartner rund um die Uhr. Sie gewährleistet die ärztliche Sofortversorgung, den Einsatz von Notarzt- und Krankenwagen und macht damit eine umgehende medizinische Betreuung von Passagieren am Boden und in der Luft wie auch von Mitarbeitern, welche während der Ausübung ihrer Tätigkeit erkranken oder einen Unfall erleiden, möglich.

In der Notfallambulanz („Flughafenklinik“) werden jährlich (365 Tage im Jahr und 24 Stunden am Tag) bis zu 36000 Patienten betreut. Drei festangestellte Notfallmediziner sowie 14 externe Fachärzte alle mit der Zusatzausbildung zum Leitenden Notarzt - sichern gemeinsam mit einem Team von zehn Krankenschwestern und -pflegern sowie Arzthelferinnen die notfallmedizinische Versorgung rund um die Uhr.

Die Aufgaben der Notfallambulanz sind vielfältig:
- allgemein- und notfallmedizinische Versorgung aller Fraportund Airlinemitarbeiter

- allgemein- und notfallmedizinische Versorgung von Passagieren, Besuchern, Frachtangestellten, Mitarbeitern und Gästen der Hotels

- Notärztliche Versorgung des Standorts Flughafen und - nach Anfrage - auch der Nachbargemeinden, Autobahnen, der USAirbase und der Stadt Frankfurt

- Überwachung der Hygiene- und Sanitätsvorschriften sowie Seuchenabwehr (Sanitätsflughafen). Die Notfallambulanz fungiert als Außenstelle des Stadtgesundheitsamtes Frankfurt. Alle Ärzte der Notfallambulanz sind als Amtsärzte für den Bereich des Flughafens bestallt

- Impfungen und Impfberatungen - zugelassene Gelbfieberimpfstelle

- Medizinische Untersuchungen im Auftrag von Behörden (z.B. Polizei, Bundesgrenzschutz, Zoll), Airlines, Spezialuntersuchungen (z.B. Taucher)

- notärztliche Versorgung der Asylbewerber

- Organisation der medizinischen Katastrophenabwehr.

An Räumlichkeiten stehen zur Verfügung: Röntgenbereich, Intensivstation (Abb. 1) und Schockraum, zwei OP-Einheiten (Abb. 2) sowie fachspezifische Behandlungskabinen (z.B. Augen), ein medizinischer Quarantänebereich mit Ruheraum sowie externe Labormöglichkeiten.

\section{Der Rettungsdienst}

Der Rettungsdienst der FraportAG sichert nicht nur das Betriebsgelände, sondern auch die umliegenden Autobahnen sowie Nachbargemeinden bei Anforderung durch die zuständigen Rettungsleitstellen. Dem Rettungsdienst - einschließlich Behindertentransportservice - sind mehr als 30 Rettungsassistenten und -sanitäter in drei Schichten zugeteilt. Die überwiegende Anzahl der Mitarbeiter im Rettungsdienst ist zum Organisatorischen Leiter ausgebildet und bildet im Schadensfall mit dem Leitenden Notarzt und 
dem Technischen Einsatzleiter der Feuerwehr die technische Einsatzleitung (TEL). Mit durchschnittlichen Eingreifzeiten - d.h. Rettungspersonal vor Ort am Patienten - von nur vier Minuten unterschreitet der Rettungsdienst den vorgegebenen Zeitraum von maximal zehn Minuten deutlich (Tab. 1, Abb. 3).

Insgesamt besteht der Fuhrpark des Rettungsdienstes aus sechs Rettungswagen, welche alle als Notarztwagen ausgerüstet sind (Abb. 4). Zusätzlich verfügt der Rettungsdienst noch über diverse Fahrzeuge wie zum Beispiel Hubwagen, Einsatzfahrzeug für den Organisatorischen Leiter. Bei schweren Notfällen steht zusätzlich ein Gerätewagen - Allrad mit Anhänger, GW-Rett (Abb. 5) zur Katastrophenabwehr zur Verfügung. Hier wird das gesamte Material zur Erstversorgung von circa 50 bis 100 Verletzten/Erkrankten gelagert.

Im Jahr 2000 absolvierte der Rettungsdienst insgesamt 22450 Einsätze, darunter allein 12000 Krankentransporte und 740 Notarztwageneinsätze.

\section{Gefahren durch Infektions- krankheiten}

Ein Großflughafen wie Frankfurt am Main kann die Eintrittspforte für Infektionskrankheiten jeglicher Art sein. Bekannte Infektionskrankheiten (z.B. Masern, Windpocken, Röteln, Polio, Cholera, Meningitis, SARS usw.) können für den Flughafen ein Problem bedeuten. Beispielsweise kann ein Ausbruch von Masern bei Asylbewerbern, aus Ländern in denen Masern nicht oder nur selten vorkommt, durchaus zu erheblichen medizinischen Problemen führen. Auch im Zeitalter von modernen Impfverfahren muss mit Epidemien gerechnet werden. Eine zunehmende Impfmüdigkeit - am Beispiel des Masernausbruchs in Italien kann diese Entwicklung noch fördern.

Ein großes Problem stellen hochkontagiöse lebensbedrohliche Erkrankungen dar. Es handelt sich dabei um verschiedene durch Viren ausgelöste hämorrhagische Fieber (z.B. Ebola, Lassa, Marburg, KrimKongo, usw.) sowie die Lungenpest und Infektionen durch Orthopoxviren. All diesen Infektionen ist gemeinsam, dass sie von Mensch zu Mensch übertragen werden können und eine hohe Mortalitätsrate haben. Entscheidend ist das zeitnahe Ergreifen aller notwendigen Maßnahmen bei Erhebung der Verdachtsdiagnose als wesentliche Voraussetzung für ein adäquates Management der lebensbedrohlichen Infektionskrankheiten.

\section{Wer stellt den Verdacht auf eine Infektionskrankheit?}

Wenn sich ein Arzt an Bord befindet, äußert dieser einen ersten Verdacht. Sollte sich kein Arzt im Flugzeug befinden, informiert die Crew über den Kapitän die zuständige Bodenstation. Von hier aus wird veranlasst, dass das Luftfahrzeug auf eine entlegene Abstellposition geleitet wird und keiner das Flugzeug verlässt. Die Luftverkehrsgesellschaft wird sich schnellstmöglich mit dem diensthabenden Arzt der Medizinischen Dienste in Verbindung setzen.

\section{Was veranlasst der dienst- habende Arzt?}

Nach Prüfung der Lage vor Ort unter Eigenschutz durch entsprechende Schutzanzüge - und Erhebung einer Verdachtsdiagnose wird das Flugzeug gegebenenfalls unter Quarantäne gestellt. Der Bereitschaftsdienst des Stadtgesundheitsamtes und die Notaufnahme der Uniklinik Frankfurt (diensthabender Infektiologe und eventuell Intensivstation) werden informiert, gleichzeitig wird das Kompetenzzentrum des Stadtgesundheitsamtes eingeschaltet. Alle Kontaktpersonen werden ermittelt und registriert, gegebenenfalls erfolgt eine notfallmäßige Behandlung des oder der Erkrankten. Die medizinischen Maßnahmen werden in enger Absprache mit dem verantwortlichen Stadtgesundheitsamt durchgeführt. Dieses legt den Umfang der seuchenhygienischen Maßnahmen fest und überwacht die Durchführung.

Sobald die Isolierstation in der Klinik eingerichtet ist, werden die Erkrankten umgehend mittels Sondertransport dorthin verlegt. Es han-

\section{Abb. 3 Zeit vom Notruf bis zum Eintref- fen am Einsatzort im Jahr 2002}

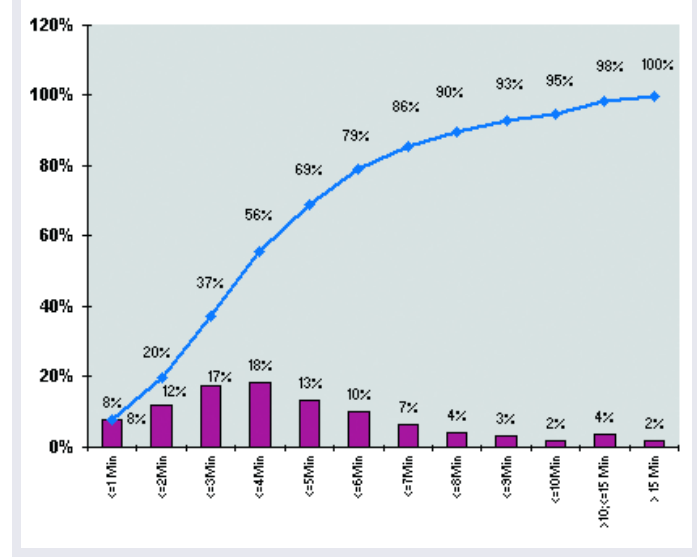

delt sich hierbei um ein Sonderfahrzeug der Berufsfeuerwehr mit ausgebildetem Personal und entsprechender Schutzausrüstung, das heißt Mund- und Augenschutz, Handschuhe und Schutzanzüge. Im Anschluss an den Transport erfolgt eine sachgerechte Desinfektion.

Dieser Ablauf gilt für den Idealfall. Im Idealfall befindet sich das Flugzeug auf Außenposition, keiner verlässt das Luftfahrzeug, Kranke und Kontaktpersonen können direkt in die entsprechenden Kliniken gebracht werden.

\section{Was passiert, falls der Kranke} bereits ausgestiegen ist?

Falls der Kranke bereits ausgestiegen ist, oder gar selbst zu Fuß zur Notfallambulanz kommt oder gebracht wird, wird die Quarantäne-

\section{Tab. 1 Eingreifzeiten des Rettungs- dienstes im Jahr 2003}

\begin{tabular}{|c|c|c|c|}
\hline Ankunft & Einsätze & Anteil & $\begin{array}{c}\text { Anteile } \\
\text { kum. }\end{array}$ \\
\hline$\leq 1 \mathrm{Min}$ & 268 & $8 \%$ & $8 \%$ \\
\hline$\leq 2 \mathrm{Min}$ & 394 & $12 \%$ & $20 \%$ \\
\hline$\leq 3 \mathrm{Min}$ & 582 & $17 \%$ & $37 \%$ \\
\hline$\leq 4 \mathrm{Min}$ & 618 & $18 \%$ & $56 \%$ \\
\hline$\leq 5 \mathrm{Min}$ & 450 & $13 \%$ & $69 \%$ \\
\hline$\leq 6 \mathrm{Min}$ & 333 & $10 \%$ & $79 \%$ \\
\hline$\leq 7 \mathrm{Min}$ & 218 & $7 \%$ & $86 \%$ \\
\hline$\leq 8 \mathrm{Min}$ & 137 & $4 \%$ & $90 \%$ \\
\hline$\leq 9 \mathrm{Min}$ & 101 & $3 \%$ & $93 \%$ \\
\hline$\leq 10 \mathrm{Min}$ & 67 & $2 \%$ & $95 \%$ \\
\hline$>10 ; \leq 15 \mathrm{Min}$ & 123 & $4 \%$ & $98 \%$ \\
\hline$\geq 15 \mathrm{Min}$ & 54 & $2 \%$ & $100 \%$ \\
\hline$\sum$ & 3345 & $100 \%$ & \\
\hline
\end{tabular}



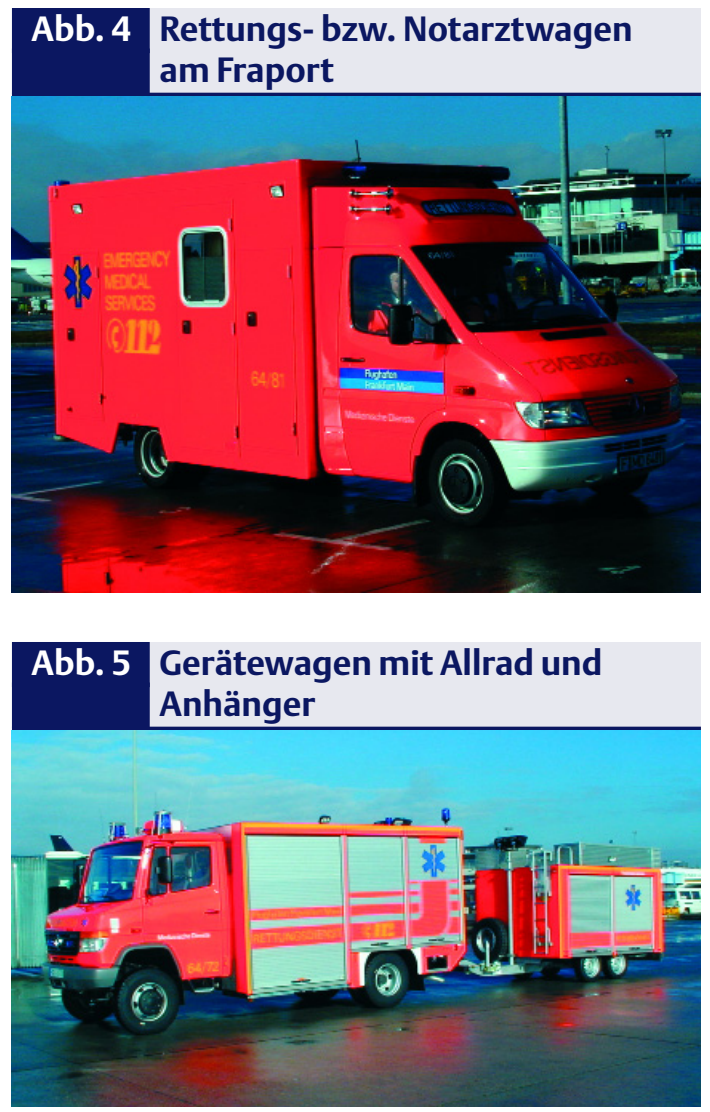

abteilung der medizinischen Dienste aktiviert. Hier können auch infektiös Erkrankte unter optimierten Bedingungen kurzfristig unter entsprechenden Schutzmaßnahmen so lange betreut werden, bis ein geeigneter Abtransport erfolgen kann.

Leider ist es nicht immer möglich, Erkrankte im Anfangsstadium zu erfassen und einer medizinischen Versorgung zuzuführen. Passagiere können - falls sie sich nicht melden, obwohl sie erkrankt sind - den Flughafen normal verlassen und am Heimatort ein Krankenhaus aufsuchen. Derartige Fälle sind zum Beispiel mit Lassa-Fieber bereits vorgekommen. In solchen Fällen ist es extrem wichtig, den Weg des Kranken zu verfolgen und alle denkbaren Kontaktpersonen zu ermitteln, sowie breitgefächerte Desinfektionsmaßnahmen durchzuführen.

Organisatorisch ist der Frankfurter Flughafen für den Umgang mit hochinfektiösen Krankheiten gerüstet. Im Zeitalter der Globalisierung muss jedoch damit gerechnet werden, dass es immer wieder neue Infektionskrankheiten geben wird, die sich nicht mehr als streng regionales Problem in fernen Ländern verhalten. Diese Herausforderungen nimmt der Flughafen Frankfurt und das Gesundheitsamt Frankfurt/Kompetenzzentrum Frankfurt an und wird bestmöglichst darauf reagieren.

Massenanfall von Verletzten

Ist der Frankfurter Flughafen aus Sicht der Feuerwehr und des Rettungsdienstes ausreichend gerüstet?

Fraport hat in enger Abstimmung mit den Behörden und Kooperationspartnern die so genannten BANOT (Betriebsanweisung Notfälle) erarbeitet, welche differenziert die unterschiedlichsten Schadensereignisse berücksichtigt. Darüber hinaus verfügt der Flughafen über qualifiziertes Personal und hochmodernes Gerät im Bereich der Flughafenfeuerwehr und des Rettungsdienstes.

\section{Gibt es Katastrophenschutz-} pläne?

Für Fraport und die am Flughafen ansässigen Firmen ist im Falle von Katastrophen die BANOT eine verbindliche Regelung. Außerhalb des Flughafenbereiches, das heißt An- und Abflugschneisen, liegt die Verantwortung bei den Städten und Gemeinden.

\section{Was passiert, wenn ein Großraumflugzeug ins Terminal 1 oder 2 stürzt?}

Auch hier greifen die Pläne der BANOT, das bedeutet „Großalarm“ auch für die Hilfskräfte der umliegenden Gemeinden. Die Steuerung des Großalarms erfolgt über die Branddirektion Frankfurt.

Was passiert, wenn ein Großraumflugzeug auf die Autobahn stürzt?

Ein solcher Absturz liegt im Verantwortungsbereich der zuständigen Gemeinden, zum Beispiel der Stadt Frankfurt, Offenbach oder Mainz.

Fraport wird aber - in Abhängigkeit seiner Ressourcen - immer unterstützend tätig werden.

Betrachtet man die unterschiedlichen Szenarien innerhalb des Flughafenbereiches und insbesondere außerhalb des Verantwortungsbereiches von Fraport, so müssen dennoch einige Fragen an die Verantwortlichen gestellt werden:

1. Gibt es Absprachen der Hilfeleistungen?

2. Werden Szenarien interdisziplinär regelmäßig geübt?

3. Werden Übungen /Planspiele regelmäßig durchgeführt?

4. Werden Übungen interdisziplinär ausgewertet?

5. Gibt es eine überregionale Behörde, welche übergreifend koordiniert?

Bei der Vielfältigkeit der Verantwortung und der unterschiedlichen Zuständigkeiten auf politischer wie auch auf kommunaler Ebene muss die Frage nach einer „übergeordneten“, koordinierenden Stelle erlaubt sein - losgelöst von Eitelkeiten und Abteilungsegoismen, ausschließlich orientiert am Wohle der Patienten.

Die Fraport AG wird - wie bisher auch - ihr Know-how auf Anfrage kostenfrei zur Verfügung stellen.

\section{Summary}

Fraport AG, the operator of Frankfurt Airport, is well prepared in regard to medical treatment in case of a disaster or emergency event. Nonetheless, all responsible persons (government agencies, politicians, cities and communities) must know that Fraport or the Fire Control Center or another medical organization could not handle a disaster (airplane crash with 100 seriously injured persons) on their own. The politicians must ensure mutual training and cooperation and see to it that there is an end to reducing staff and transportation capacities.

The surrounding cities and communities must realistically deal with the possibility of a disaster. This calls for preparation of disaster plans, exercises and continuous review or updating of the plans.

\section{Anschrift des Verfassers}

Dr. med. Walter Gaber Leiter Medizinische Dienste Fraport AG

Flughafen Frankfurt w.Gaber@fraport.de 